\title{
Characterization of main components in Xiao'er Xiaoji Zhike oral liquid by UPLC-MS and their taste evaluation
}

\author{
Chunying WU ${ }^{1}$, Shuyu WANG ${ }^{1}, \mathrm{Ou} \mathrm{TAO}^{1}$, Xueyan $\mathrm{ZHAN}^{1 *}$ (D)
}

\begin{abstract}
This paper provided a method for determining the potential quality markers in Xiao'er Xiaoji Zhike oral liquid (XXZOL) based on the concordant tastes of compounds with their respective originated Chinese medicinal pieces. UPLC-Q-ExactiveOrbitrap-MS technology was used to identify the main chemical constituents in XXZOL. The electronic tongue collected the electronic responses of the sour, bitter, sweet, pungent, and salty sample solutions, and the discriminant factor analysis (DFA) model was established to recognize the taste characteristics of 23 liquid samples. Fifteen high content ingredients in XXZOL were identified by UPLC-MS, and the established DFA model recognized their respective tastes. The accurate recognition rate of the DFA model was $73.33 \%$, the false rate was $6.67 \%$, and the unrecognized rate was $20 \%$. The concordance rate of their authentic tastes with the tastes of their respective originated Chinese medicinal pieces was $78.57 \%$. Trigonelline, malic acid, citric acid, and caffeic acid were the potential sour material bases of XXZOL. Mannitol was the potential sweet material basis of XXZOL. L-phenylalanine, sinapine, forsythoside I, pinoresinol-4-O- $\beta$-D-glucopyranoside, naringin, and neohesperidin were the potential bitter material bases of XXZOL. So the above 11 main compounds were the potential quality markers of XXZOL.
\end{abstract}

Keywords: Xiaoer Xiaoji Zhike oral liquid; UPLC-Q-Exactive-Orbitrap-MS; electronic tongue; potential quality marker.

Practical Application: A method for determining the potential quality markers in Xiaoer Xiaoji Zhike oral liquid based on the concordant tastes of compounds with their respective originated Chinese medicinal pieces.

\section{Introduction}

The electronic senses electronically reproduce the responses similar to the five senses presenting in human beings, such as electronic eye (Orlandi et al., 2018), electronic ear, electronic skin (Zhang et al., 2020b), electronic tongue (e-tongue) (Toko,1998) and electronic nose (Mohd Ali et al., 2020). These electronic sensory technologies can be used alone (Gan et al., 2019; Pascual et al., 2018; Nategh et al., 2021) or combined (Orlandi et al., 2019; Banerjee et al., 2019; Xu et al., 2019) to achieve experimental purposes. The e-tongue is a kind of liquid analytical instrument consisting of three parts, including sensor array, signal acquisition system, pattern recognition system (Toko, 1998), and taste sensations of the sensor array can usually be classified into sweet, sour, salty, bitter, and umami. Sensor array imitates the taste cells in taste buds that interact with the flavorful substance and transmit the taste information to nerve fibers and the relevant areas in the central nervous system (CNS) (Pandurangan \& Hwang, 2015). The non-specific and poorly selective sensors in the sensory array are immersed into a sample solution and acquire a global characteristic response signal of the substances in the sample (Jiang et al., 2018). Then the corresponding signals are sent to a signal processing system equivalent to CNS, which can analyze the acquired signals using appropriate pattern recognition methods. The e-tongue can realize the qualitative and quantitative analysis of simple or complex liquid samples and obtain the results reflecting the taste characteristics of samples (Legin et al., 2000). Based on the working principle of the sensor array, the e-tongue can be classified into electrochemical (Wei et al., 2018; Ciosek \& Wróblewski, 2011), optical and enzymatic e-tongue. Besides the advantages of low cost and simple operation, sensitivity, reliability, and robustness, e-tongue has its particular advantage to analyze the taste of some toxic substances (Wadehra \& Patil, 2016). The e-tongue has been widely used in different fields of food (Wadehra \& Patil, 2016; Ghasemi-Varnamkhasti et al., 2018; Peris \& Escuder-Gilabert, 2016), medicine (Wasilewski et al., 2019; Guedes et al., 2021), or environmental detection (Shimizu et al., 2019; Magro et al., 2018). The e-tongue system has good applications in quality monitoring of Chinese medicinal pieces (Shi et al., 2018), origins identification (Wu et al., 2018) and harvest period determination of herbal medicines, and the evaluation of the pharmaceutical process of traditional Chinese medicine (TCM) (Bi et al., 2020), such as correcting or masking the taste of the decoction from TCM (Lin et al., 2016; Feng et al., 2019).

Xiaoer Xiaoji Zhike oral liquid (XXZOL), a prescription preparation of TCM, with the effect of clearing heat in lungs, relieving cough due to lung heat, and helping digestion, usually is applied to treat children's respiratory diseases such as mycoplasma pneumonia (Zhang et al., 2020a), asthma (Zhou et al., 2020) and cough (Liang et al., 2018). Because of its safety and definite effects, the market sales of XXZOL have been increasing steadily. There are 10 Chinese medicinal pieces in the prescription of XXZOL 
according to their taste deployment to reduce the toxicity and bias of a single Chinese medicinal piece and achieve the overall synergy of all Chinese medicinal pieces. This prescription is the compatibility of stir-baked Crataegi Fructus, Arecae Semen, honeyed Eriobotryae Folium, Trichosanthis Fructus, Platycodonis Radix, Forsythiae Fructus, Aurantii Fructus Immaturus, stir-baked Raphani Semen, stir-baked Descurainiae Semen Lepidii Semen, and Cicadae Periostracum (China, 2020). The taste of a single herbal decoction or a prescription preparation decoction is stable only when the type and quantity of the chemical compounds in the sample solution do not alter. According to the main tastes of Chinese medicinal pieces from the prescription, to understand the recipe-construction rule of XXZOL, high content components in XXZOL were identified. Their tastes were recognized respectively by the e-tongue system. Comparing with the main tastes of Chinese medicinal pieces from the prescription, components with consistent tastes are more likely to be the material bases of XXZOL.

In our work, the UPLC-Q-Exactive-Orbitrap-MS platform was used to analyze the chemical constituents of XXZOL, and the main components were identified by the high-resolution mass spectrometry (MS) technology and control substances. The e-tongue collected the sour, bitter, sweet, pungent, and salty samples, which were $1.0 \mathrm{mg} / \mathrm{mL}$ solutions of the reference components with above tastes, and the discriminant factor analysis (DFA) model to recognize the taste characteristics of liquid samples was established to predict the taste of every main component in XXZOL. Considering the main tastes of Chinese medicinal pieces from XXZOL, we obtained several main components in XXZOL with similar tastes, which were likely to be the important quality markers of the prescription, providing a basis for the determination of the quality markers of XXZOL.

\section{Materials and methods}

\subsection{Experimental materials}

Acesulfame potassium, andrographolide, berberine, citric acid, fructose, gallic acid, gentiopicroside, glycine, oxymatrine, piperine, saccharin, salicin, sodium chloride, sucrose, and zingerone were purchased from Shanghai Yuanye Bio-Technology
Co., Ltd (Shanghai, China). Liquiritin and potassium iodide were purchased from Shanghai Macklin Biochemical Co., Ltd (Shanghai, China). Citric acid monohydrate was purchased from Xilong Scientific Co., Ltd (Guangdong, China). Potassium chloride was purchased from Beijing Chemical Works (Beijing, China). Nonivamide was purchased from Shanghai Aladdin Biochemical Technology Co., Ltd (Shanghai, China). Stevioside was purchased from Chengdu Push Bio-Technology Co., Ltd (Chengdu, Sichuan province, China). Caffeic acid, forsythoside A, forsythoside E, forsythoside I, L-phenylalanine, malic acid, maltose monohydrate, naringin, neohesperidin, pinoresinol-4$\mathrm{O}-\beta$-D-glucopyranoside, sinapine, synephrine, and trigonelline were purchased from Chengdu DeSiTe Biological Technology Co., Ltd (Chengdu, Sichuan province, China). Mannitol was purchased from Tianjin Fuchen Chemical Reagent Co., Ltd (Tianjin, China). The information of the reference components used for the e-tongue experiments was shown in Table 1, and their contents were greater than or equal to $98 \%$. MS grade acetonitrile and formic acid were purchased from Thermo Fisher Scientific (USA). Analytical grade ethanol was purchased from Shanghai Rhawn Chemical Technology Co., Ltd (Shanghai, China).

XXZOL was purchased from Lunan Hope Pharmaceutical Co., Ltd (Linyi, Shangdong province, China). Take the oral liquid $0.25 \mathrm{~mL}$ precisely to $10 \mathrm{~mL}$ volumetric flask, water was added to the mark. Then, the solution was filtered with a membrane filter $(0.22 \mu \mathrm{m})$ to collect the successive filtrate as the sample solution.

The powder $(25 \mathrm{mg}$ ) of each reference component was dissolved in $25 \mathrm{~mL}$ of $50 \% \mathrm{v} / \mathrm{v}$ aqueous ethanol with ultrasonication at room temperature for 10 minutes. The obtained solutions were collected for electronic tongue analysis.

\subsection{LC-MS/MS analytical conditions}

LC-MS/MS was carried out on a UPLC-Q-ExactiveOrbitrap-MS platform (Thermo Fisher Scientific, USA) with a Waters ACQUITY UPLC HSS T3 Column $(100 \mathrm{~mm} \times 2.1 \mathrm{~mm}$, $1.8 \mu \mathrm{m})$. The mobile phases consisted of acetonitrile (A) and $0.1 \%$ aqueous formic acid $(\mathrm{v} / \mathrm{v})(\mathrm{B})$ using gradient elutions of $0-2 \% \mathrm{~A}$ at $0-10 \mathrm{~min}, 2-7 \% \mathrm{~A}$ at $10-20 \mathrm{~min}, 7-14 \% \mathrm{~A}$ at $20-30 \mathrm{~min}$, $14-14.5 \% \mathrm{~A}$ at $30-32 \mathrm{~min}, 14.5-15 \% \mathrm{~A}$ at $32-37 \mathrm{~min}, 15-15.5 \mathrm{~A}$ at $37-40 \mathrm{~min}, 15.5-17 \% \mathrm{~A}$ at $40-45 \mathrm{~min}, 17-24 \% \mathrm{~A}$ at $45-53 \mathrm{~min}$,

Table 1. Reference components and their taste for electronic tongue tests.

\begin{tabular}{lll}
\hline \multicolumn{1}{c}{ Taste } & Type of reference component & \multicolumn{1}{c}{ Reference component (No.) } \\
\hline Sour & Organic acid & Citric acid (SO-1), citric acid monohydrate (SO-2), gallic acid (SO-3), malic acid (SO-4) \\
& Alkaloid & Berberine (B-1), oxymatrine (B-2) \\
& Glycoside & Salicin (B-3) \\
Sweet & Terpenoid & Andrographolide (B-4), gentiopicroside (B-5) \\
& Amino acid & Glycine (SW-1) \\
& Carbohydrate & Fructose (SW-2), maltose monohydrate (SW-3), sucrose (SW-4) \\
& Flavonoid & Liquiritin (SW-5) \\
& Terpenoid & Stevioside (SW-6) \\
Pungent & Other & Acesulfame potassium (SW-7), saccharin (SW-8) \\
& Alkaloid & Nonivamide (P-1), piperine (P-2) \\
Salty & Aromatic hydrocarbon & Zingerone (P-3) \\
\hline
\end{tabular}


$24-31 \% \mathrm{~A}$ at $53-58 \mathrm{~min}, 31-37 \% \mathrm{~A}$ at $58-59 \mathrm{~min}, 37-38 \% \mathrm{~A}$ at $59-63 \mathrm{~min}, 38-55 \% \mathrm{~A}$ at $63-64 \mathrm{~min}, 55-58 \% \mathrm{~A}$ at $64-69 \mathrm{~min}$, $58-100 \% \mathrm{~A}$ at $69-70 \mathrm{~min}, 100 \% \mathrm{~A}$ at $70-75 \mathrm{~min}$. The mobile phase flow rate was $0.4 \mathrm{~mL} / \mathrm{min}$, and the column temperature was maintained at $30{ }^{\circ} \mathrm{C}$. The injection volume was $5 \mu \mathrm{L}$. The ESI-MS was performed in both positive and negative modes with the source settings as follows: spray voltage, $3800 \mathrm{~V}(+)$, $3200 \mathrm{~V}(-)$; auxiliary gas flow rate, 15 arb; sheath gas flow rate, 35 arb; capillary temperature, $350{ }^{\circ} \mathrm{C}$; scan range, $\mathrm{m} / \mathrm{z}$ 100-1500; collision dissociation energy, $30 \mathrm{eV}, 40 \mathrm{eV}, 50 \mathrm{eV}$. MS data was collected with Thermo Xcalibur software.

\subsection{The electrochemical e-tongue device and data collected parameters}

The ASTREE e-tongue (Alpha M.O.S., France) equipped with the fifth set of sensor systems was used, and the sensor system had 7 electrochemical sensors (SRS, GPS, STS, UMS, SPS, SWS, BRS) and an Ag/AgCl reference electrode. After 7 electrochemical sensors were activated and calibrated, these sensors were put into a liquid sample to collect the data, including taste characteristics of the liquid sample. The parameters for the e-tongue data collected were as follows, the data acquisition time $120 \mathrm{~s}$, acquisition period $1.0 \mathrm{~s}$, acquisition delay $0 \mathrm{~s}$, stirring rate $1.0 \mathrm{r} \cdot \mathrm{s}^{-1}$. Nine replicates for each sample, only the average of the $100-120 \mathrm{~s}$ data of the last 3 replicates were kept as 3 results for each sample to ensure the stability of the sensor response values, indicating that the sensor responses were stable when the RSD value of 3 results for each sample was less than $5 \%$.

\subsection{Data processing}

The Xcalibur 4.0 software (Thermo Fisher Scientific, USA) was used to calculate the high-resolution accurate mass of the compounds. Based on error less than $5 \mathrm{ppm}$ and MS/MS fragment matching, we identified main compounds in XXZOL, and the identified substances were used to verify the identified results.

Principal component analysis (PCA) and DFA were used to recognize the taste characteristics of liquid samples of reference components and establish the classification model of five kinds of tastes. The taste of every main component in XXZOL was discriminated by the FDA model.

\section{Results and discussion}

\subsection{Identification of main chemical compounds in XXZOL}

The negative and positive total ion chromatograms of XXZOL were shown in Figure 1. Due to their high responses in the mass spectrometer and UV spectrometer, high content compounds in XXZOL were focused on, and 15 high content compounds in XXZOL were chosen considering the compounds' solubility in $50 \%$ ethanol. The high-resolution accurate mass and major MS/MS fragments of the above 15 main compounds were presented in Table 2, and the mass relative errors between the theoretical mass and measured mass of 15 main compounds were all smaller than $5 \mathrm{ppm}$, and the MS/MS fragments measured of each compound existed rationally and accorded with the MS/ MS fragments reported in the works of literature.

Table 2. 15 main components of XXZOL identified by LC-MS/MS.

\begin{tabular}{|c|c|c|c|c|c|c|c|c|}
\hline No. & $\begin{array}{l}\text { Retention } \\
\text { time }\end{array}$ & Compound & $\begin{array}{l}\text { Molecular } \\
\text { formula }\end{array}$ & Ion type & $\begin{array}{c}\text { Theoretical } \\
\mathrm{m} / \mathrm{z}\end{array}$ & $\begin{array}{c}\text { Measured } \\
\mathrm{m} / \mathrm{z}\end{array}$ & Error ppm & Major MS/MS fragments \\
\hline 1 & 0.66 & Mannitol & $\mathrm{C}_{6} \mathrm{H}_{14} \mathrm{O}_{6}$ & {$[\mathrm{M}-\mathrm{H}]^{-}$} & 181.07066 & 181.07085 & 1.05 & $\begin{array}{l}163.06062,119.03394, \\
101.02320,89.02316,85.02821 \\
73.02821,71.01254,59.01259\end{array}$ \\
\hline 2 & 0.80 & Trigonelline & $\mathrm{C}_{7} \mathrm{H}_{7} \mathrm{NO}_{2}$ & {$[\mathrm{M}+\mathrm{H}]^{+}$} & 138.05495 & 138.05490 & 0.36 & $110.06025,94.06551,92.04988$ \\
\hline 3 & 0.98 & Sucrose & $\mathrm{C}_{12} \mathrm{H}_{22} \mathrm{O}_{11}$ & {$[\mathrm{M}-\mathrm{H}]^{-}$} & 341.10784 & 341.10858 & 2.17 & $\begin{array}{l}119.03384,113.02325, \\
101.02320,89.02313,71.01256, \\
59.01260\end{array}$ \\
\hline 4 & 1.03 & Malic acid & $\mathrm{C}_{4} \mathrm{H}_{6} \mathrm{O}_{5}$ & {$[\mathrm{M}-\mathrm{H}]^{-}$} & 133.01315 & 133.01302 & 0.98 & $\begin{array}{l}\text { 133.01315, 115.00250, 89.02316, } \\
72.99179,71.01254\end{array}$ \\
\hline 5 & 1.40 & Synephrine & $\mathrm{C}_{9} \mathrm{H}_{13} \mathrm{NO}_{2}$ & {$[\mathrm{M}+\mathrm{H}]^{+}$} & 168.10191 & 168.10173 & 1.07 & $\begin{array}{l}150.09122,135.06776, \\
119.04924,91.05463\end{array}$ \\
\hline 6 & 2.12 & Citric acid & $\mathrm{C}_{6} \mathrm{H}_{8} \mathrm{O}_{7}$ & {$[\mathrm{M}-\mathrm{H}]^{-}$} & 191.01863 & 191.01889 & 1.36 & $111.00758,87.00749,85.02821$ \\
\hline 7 & 7.59 & L-phenylalanine & $\mathrm{C}_{9} \mathrm{H}_{11} \mathrm{NO}_{2}$ & {$[\mathrm{M}+\mathrm{H}]^{+}$} & 166.08626 & 166.08623 & 0.18 & $131.04921,120.08099,103.05453$ \\
\hline 8 & 21.71 & Forsythoside E & $\mathrm{C}_{20} \mathrm{H}_{30} \mathrm{O}_{12}$ & {$[\mathrm{M}-\mathrm{H}]^{-}$} & 461.16535 & 461.16635 & 2.17 & $\begin{array}{l}205.07127,143.03397, \\
135.04413,131.03394, \\
101.02319,89.02315,73.02821, \\
71.01257,59.01260\end{array}$ \\
\hline 9 & 22.32 & Caffeic acid & $\mathrm{C}_{9} \mathrm{H}_{8} \mathrm{O}_{4}$ & {$[\mathrm{M}-\mathrm{H}]^{-}$} & 179.03389 & 179.03413 & 1.34 & 135.04410 \\
\hline 10 & 25.93 & Sinapine & $\mathrm{C}_{16} \mathrm{H}_{24} \mathrm{NO}_{5}$ & {$[\mathrm{M}]^{+}$} & 310.16490 & 310.16473 & 0.55 & $251.09106,175.03885$ \\
\hline 11 & 34.36 & Forsythoside I & $\mathrm{C}_{29} \mathrm{H}_{36} \mathrm{O}_{15}$ & {$[\mathrm{M}-\mathrm{H}]^{-}$} & 623.19705 & 623.19788 & 1.33 & $179.03410,161.02353,135.04408$ \\
\hline 12 & 38.42 & Forsythoside A & $\mathrm{C}_{29} \mathrm{H}_{36} \mathrm{O}_{15}$ & {$[\mathrm{M}-\mathrm{H}]^{-}$} & 623.19705 & 623.19800 & 1.52 & $179.03423,161.02354,135.04408$ \\
\hline 13 & 39.21 & $\begin{array}{l}\text { Pinoresinol- } \\
\text { 4-O- } \beta \text {-D- } \\
\text { glucopyranoside }\end{array}$ & $\mathrm{C}_{26} \mathrm{H}_{32} \mathrm{O}_{11}$ & {$[\mathrm{M}-\mathrm{H}]^{-}$} & 519.18609 & 519.18707 & 1.89 & $357.13467,151.03914,136.01556$ \\
\hline 14 & 42.70 & Naringin & $\mathrm{C}_{27} \mathrm{H}_{32} \mathrm{O}_{14}$ & {$[\mathrm{M}-\mathrm{H}]^{-}$} & 579.17083 & 579.17081 & 0.03 & $271.06143,151.00270,119.04909$ \\
\hline 15 & 44.98 & Neohesperidin & $\mathrm{C}_{28} \mathrm{H}_{34} \mathrm{O}_{15}$ & {$[\mathrm{M}-\mathrm{H}]^{-}$} & 609.18140 & 609.18231 & 1.49 & 301.07172 \\
\hline
\end{tabular}




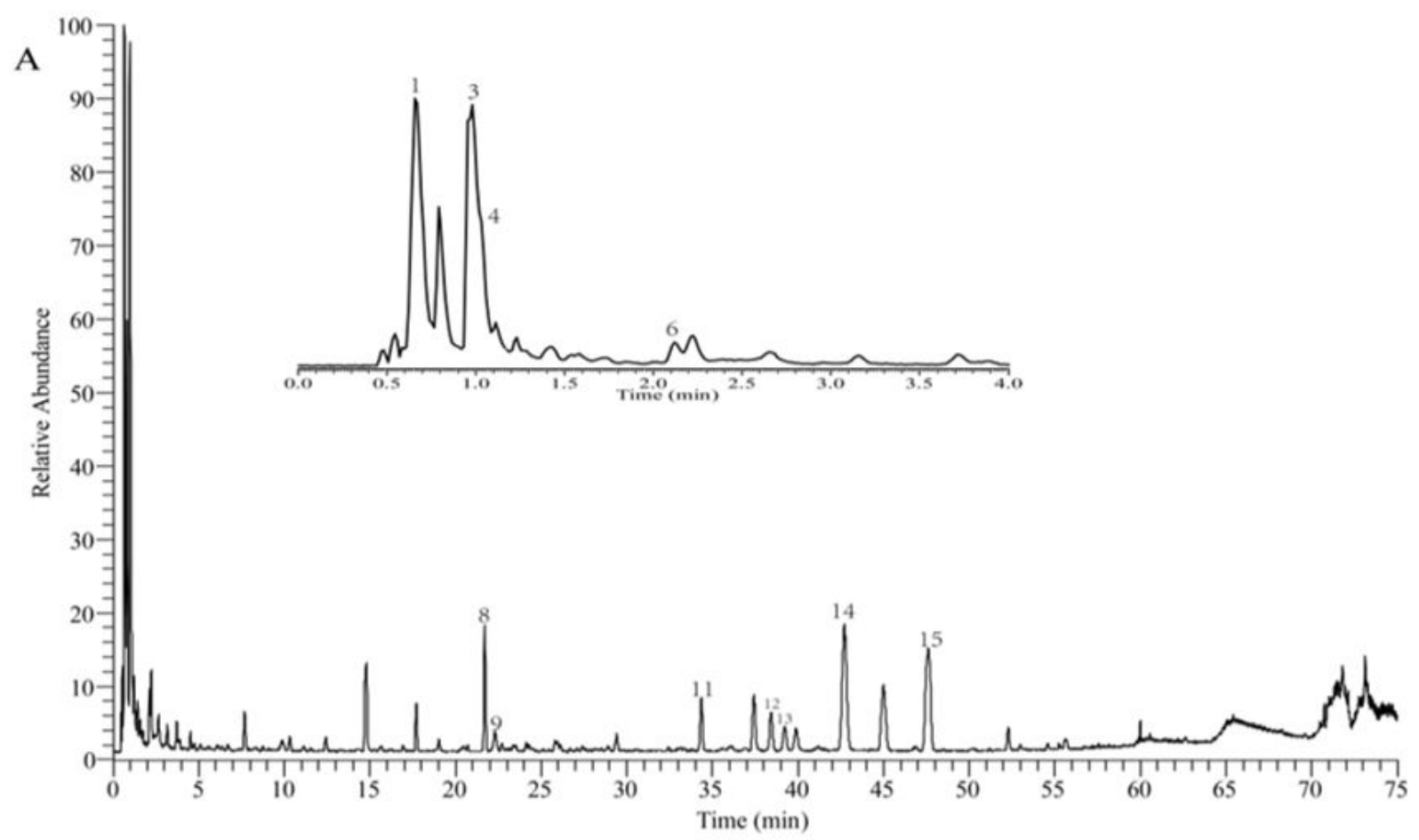

B

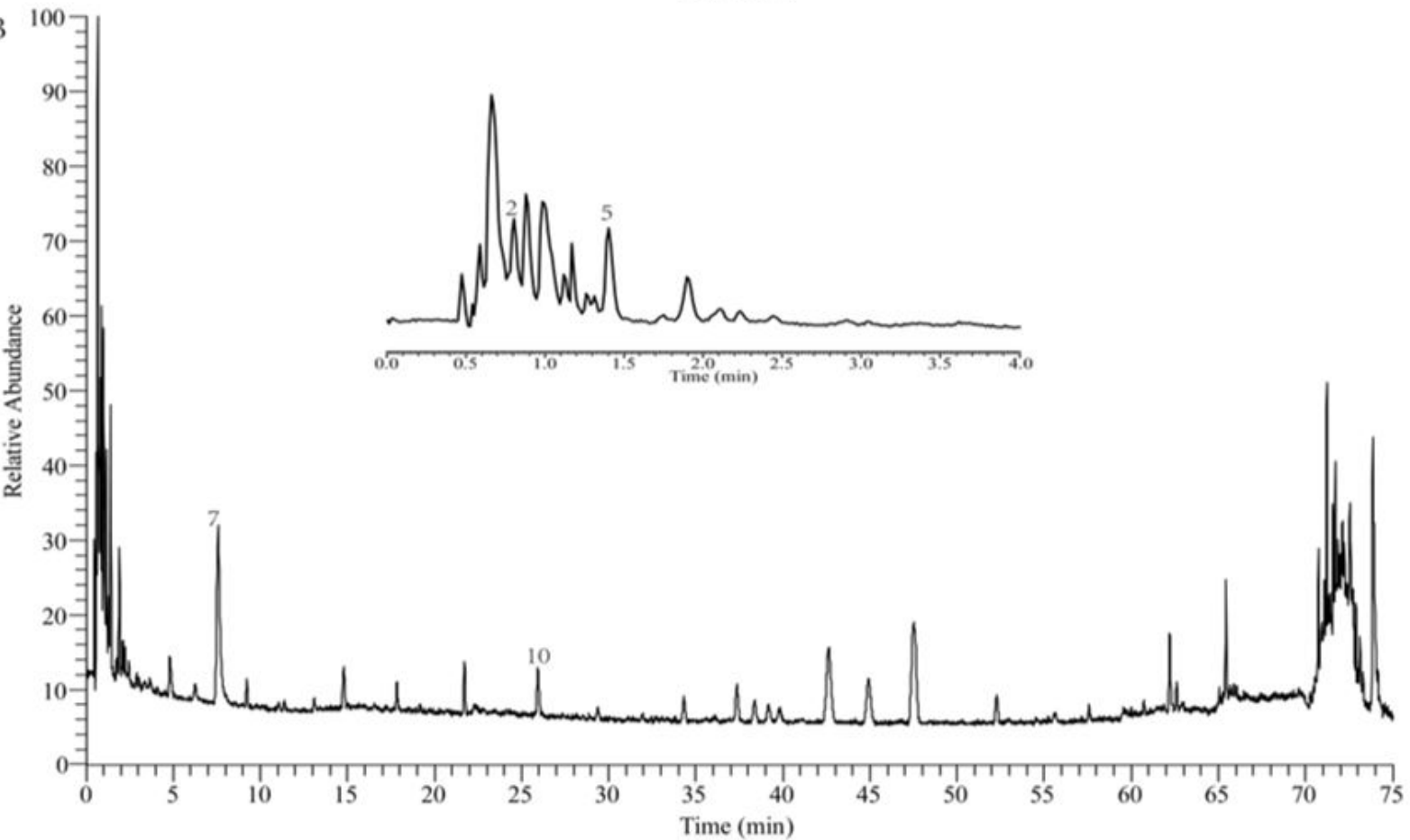

Figure 1. The negative (A) and positive (B) total ion chromatogram of XXZOL. 1-mannitol, 2-trigonelline, 3-sucrose, 4-malic acid, 5-synephrine, 6-citric acid, 7-L-phenylalanine, 8-forsythoside E, 9-caffeic acid, 10-sinapine, 11-forsythoside I, 12 -forsythoside A, 13-pinoresinol-4-O- $\beta$-Dglucopyranoside, 14-naringin, 15-neohesperidin.

Furthermore, 12 main compounds were identified with the corresponding control substances in the DAD spectrometer at the detection wavelength of $254 \mathrm{~nm}$, (A) the sample chromatogram, (B) the mixed control substances chromatogram, as shown in Figure 2, UV absorption spectrum and retention time for each compound in the sample were consistent with those in control substances.

\subsection{Taste judgments of 15 main components in XXZOL by electronic tongue}

Analysis of taste resolution ability of electronic tongue

Prepare $50 \%$ ethanol solutions with high $(8.0 \mathrm{mg} / \mathrm{mL})$, medium $(5.0 \mathrm{mg} / \mathrm{mL})$, and low $(1.0 \mathrm{mg} / \mathrm{mL})$ concentrations of 


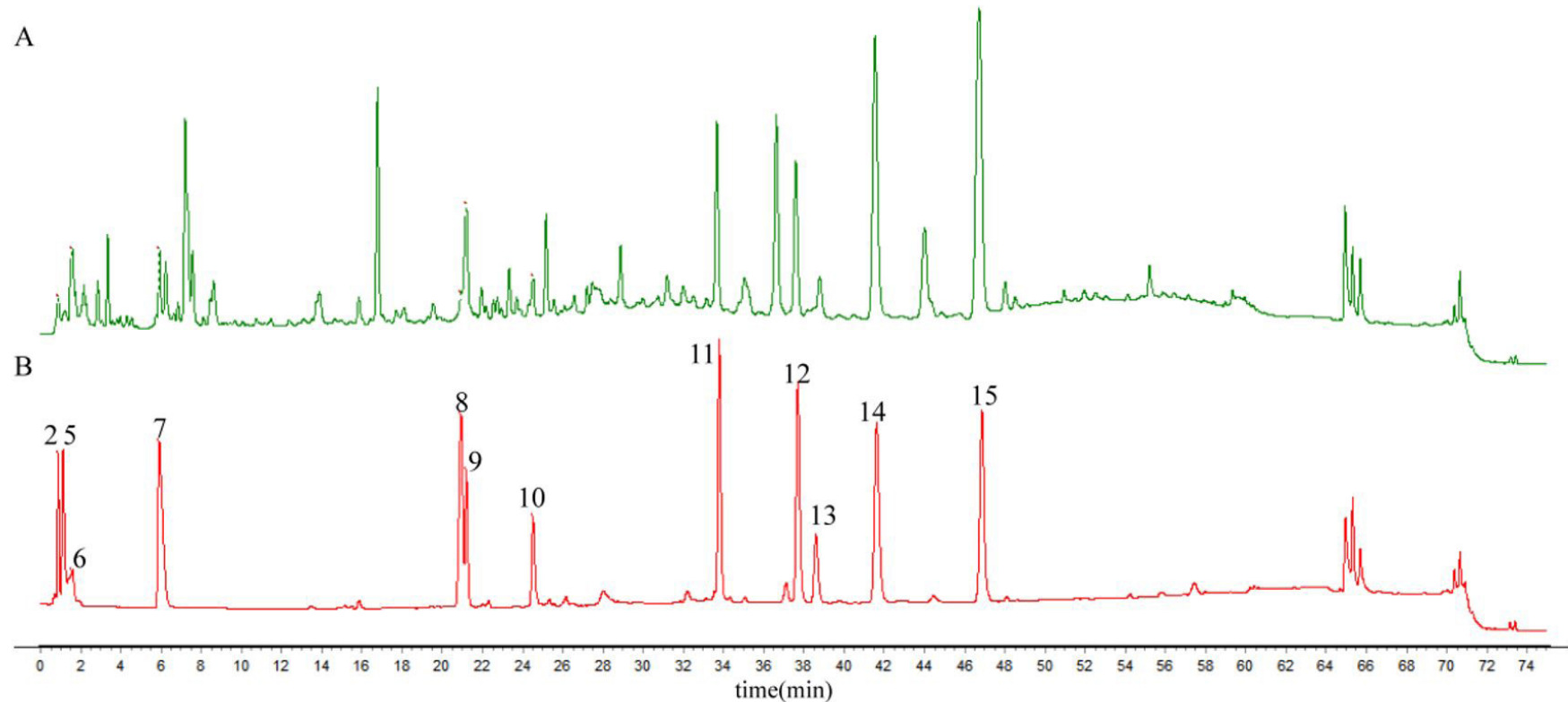

Figure 2. The sample chromatogram (A) and the mixed control substances chromatogram (B) at the detection wavelength of $254 \mathrm{~nm} .2$-trigonelline, 5-synephrine, 6-citric acid, 7-L-phenylalanine, 8-forsythoside E, 9-caffeic acid, 10-sinapine, 11-forsythoside I, 12-forsythoside A, 13-pinoresinol4-O- $\beta$-D-glucopyranoside, 14-naringin, 15-neohesperidin.

citric acid monohydrate (sour), quinine (bitter), sucrose (sweet), zingerone (pungent) and sodium chloride (salty), and collect the data of the above 15 solutions by the e-tongue, 3 replicates for each solution. PCA and DFA were applied to process the e-tongue data of 15 samples, as shown in Figure 3, 3 collected data of the same solution well-replicated, which indicated the stability of the e-tongue platform and the robustness of the e-tongue data.

In the two-dimensional PCA scatter plot of 5 reference components with different tastes, the accumulative contribution rate for the two PCs was $91.264 \%$, in which PC1 accounted for $69.075 \%$, and PC2 contributed $22.189 \%$, and five reference components with different tastes were differentiated (Figure 3A). Three different concentration samples from the same reference component had small changes in e-tongue data and were all clustered into the same category in the two-dimensional PCA plot. The results showed that the e-tongue combined with PCA could distinguish the reference components with different tastes, and concentration of the sample solution had little effect on the classification of the PCA model.

In the two-dimensional DFA scatter plot of 5 reference components with different tastes, the accumulative contribution rate with the two DFs was $97.395 \%$, in which DF1 accounted for $91.131 \%$, and DF2 contributed 6.264\%. Five reference components with different tastes were better differentiated in Figure 3B when compared with the results of the PCA model (Figure 3A), the projection distances between different concentration samples from the same reference component, i.e., the projection distances in the same group were more concentrated, which showed that concentration of sample solution also had little effect on the classification of the DFA model. The distances between groups were further increased in DFA scatter plot, indicating that the DFA model of the e-tongue data had better classification results for five reference components with different tastes than the PCA model.

The classification model of the e-tongue data could effectively distinguish the reference components with different tastes. The different concentration samples of the same reference component overlapped together in the two-dimensional PCA or DFA scatter plot, indicating that the concentration of the sample solution had little effect on the classification ability of the classification model. So $1.0 \mathrm{mg} / \mathrm{mL}$ reference component solutions were prepared in the following research, and DFA was applied to the sensor data of these solutions collected by the e-tongue platform.

\section{DFA classification model for distinguishing components with different tastes}

Prepare $1.0 \mathrm{mg} / \mathrm{mL} 50 \%$ ethanol solutions of reference components in Table 1, collect data according to the parameters of the e-tongue in section 2.3, and DFA was used to recognize the taste characteristics of reference component solutions with five different tastes.

In the two-dimensional DFA scatter plot of these reference component samples with 5 kinds of tastes, the accumulative contribution rate with the two DFs was $75.023 \%$, in which DF1 was $44.389 \%$, and DF2 was $30.634 \%$. Reference components with different tastes were differentiated, and reference components with the same taste were clustered into one category (Figure 4). The results showed that the e-tongue combined with DFA could effectively distinguish reference components with different tastes. 

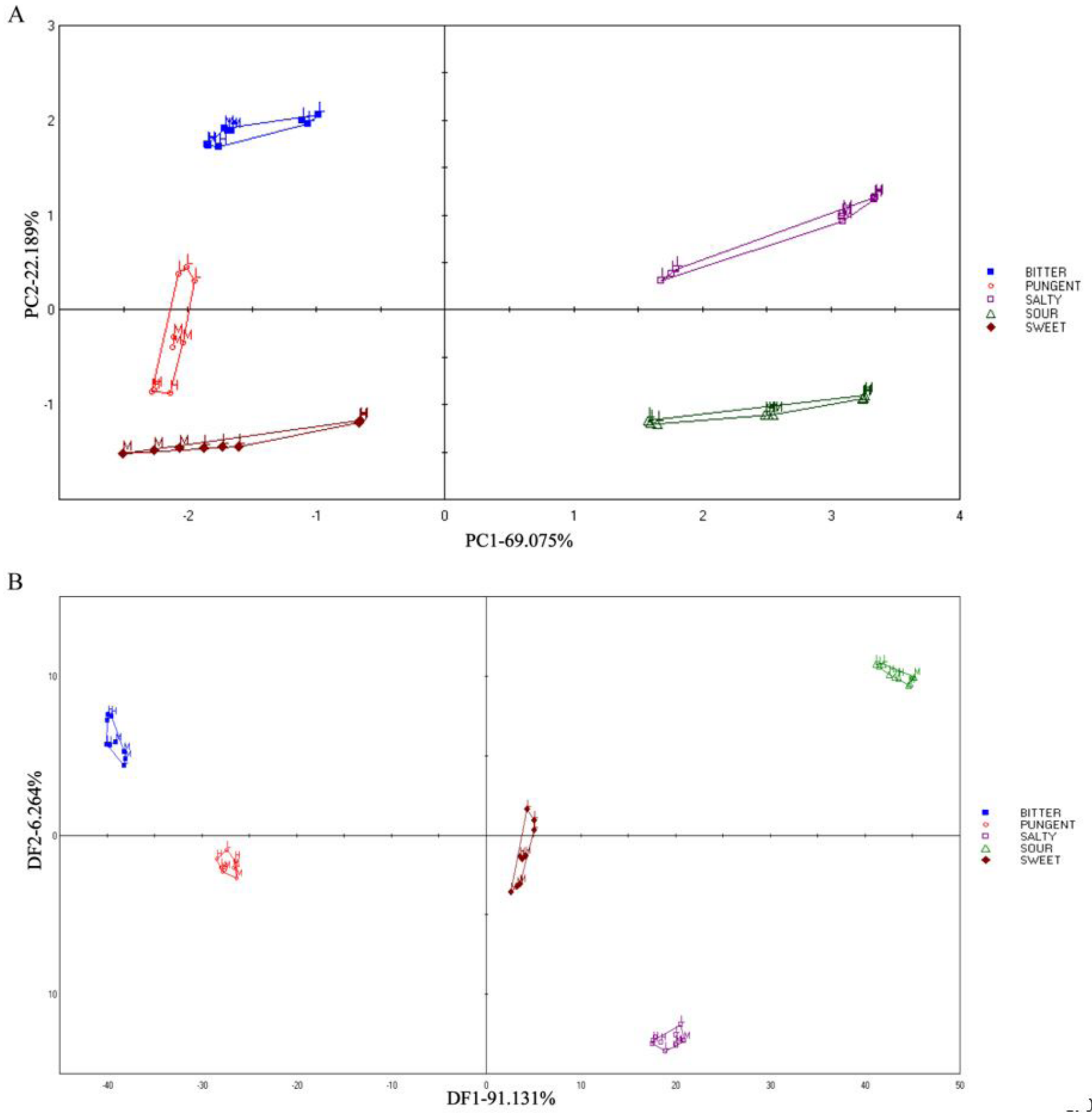

Figure 3. Two-dimensional PCA (A) and DFA (B) scatter plots of five reference components with different tastes. L-low concentration of 1.0 $\mathrm{mg} / \mathrm{mL}, \mathrm{M}$-medium concentration of $5.0 \mathrm{mg} / \mathrm{mL}, \mathrm{H}$-high concentration of $8.0 \mathrm{mg} / \mathrm{mL}$.

\section{Taste judgments of 15 main components in XXZOL}

Prepare $1.0 \mathrm{mg} / \mathrm{mL} 50 \%$ ethanol solutions of 15 main components in XXZOL. The established DFA model was used to discriminate the tastes of these solutions. The taste information of 15 main components was shown in Table 3. Authentic taste of each component was evaluated by three assessors, predicted taste of each component was judged by the DFA model of the reference components in Table 1, the originated Chinese medicinal piece was obtained by the reported works of literature, and the piece taste was obtained by the Chinese Pharmacopoeia (2020 version a).

As shown in Table 3, the tastes of 12 main components were effectively recognized by the established DFA model. Except for forsythoside I, the predicted tastes of mannitol, trigonelline, sucrose, malic acid, citric acid, L-phenylalanine, forsythoside E, caffeic acid, forsythoside A, naringin, and neohesperidin were 


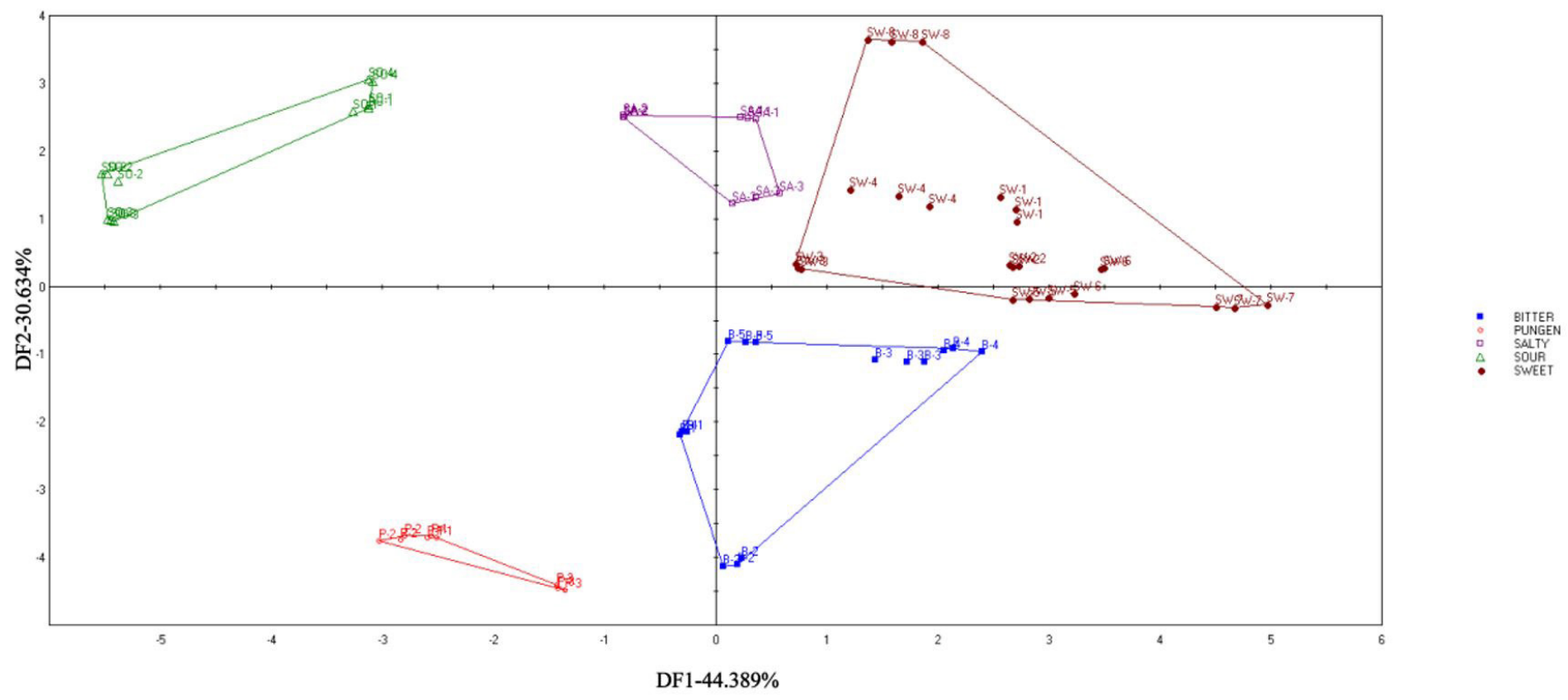

Figure 4. Two-dimensional DFA scatter plot of the reference components in Table $1(1.0 \mathrm{mg} / \mathrm{mL})$.

Table 3. The taste information of 15 main components in XXZOL.

\begin{tabular}{|c|c|c|c|c|}
\hline Compound & Authentic taste & Predicted taste & Originated Chinese medicinal piece & $\begin{array}{l}\text { Main taste of Chinese } \\
\text { medicinal piece }\end{array}$ \\
\hline Trigonelline & Sour & Sour & Trichosanthis Fructus & Sour, sweet \\
\hline Malic acid & Sour & Sour & Stir-baked Crataegi Fructus & Sour, sweet \\
\hline Synephrine & Pungent & $一^{\mathrm{a}}$ & Aurantii Fructus Immaturus & Bitter, sour \\
\hline Forsythoside E & Sweet & Sweet & Forsythiae Fructus & Bitter \\
\hline Caffeic acid & Sour & Sour & Stir-baked Crataegi Fructus & Sour, sweet \\
\hline Sinapine & Bitter & $-^{\mathrm{a}}$ & $\begin{array}{l}\text { Stir-baked Descurainiae Semen Lepidii Semen, Stir-baked } \\
\text { Raphani Semen }\end{array}$ & Bitter, pungent \\
\hline Forsythoside I & Bitter & Sweet & Forsythiae Fructus & Bitter \\
\hline Neohesperidin & Bitter & Bitter & Aurantii Fructus Immaturus & Bitter, sour \\
\hline
\end{tabular}

consistent with their authentic tastes. The accurate recognition rate for the above 15 main components was $73.33 \%$, the false rate was $6.67 \%$, and the unrecognized rate was $20 \%$. When the number and representativeness of the reference components for the established DFA model increase, the unrecognized rate will further decrease. So the e-tongue combined with classification model can be used for predicting the tastes of different components in Chinese medicinal pieces.

For XXZOL, exclude sucrose as the additive of the preparation, the remaining 14 ingredients with high responses in the mass spectrometer, were all from Chinese medicinal pieces in XXZOL.
The authentic tastes of mannitol, trigonelline, malic acid, citric acid, L-phenylalanine, caffeic acid, sinapine, forsythoside I, pinoresinol4-O- $\beta$-D-glucopyranoside, naringin, and neohesperidin were similar to the main tastes of their respective originated Chinese medicinal pieces. The concordance rate of authentic tastes of 14 compounds with the main tastes of their respective originated Chinese medicinal pieces was $78.57 \%$, the false rate was $21.43 \%$.

The originated Chinese medicinal pieces were the main herbal decoctions in the prescription of XXZOL, including Stir-baked Crataegi Fructus, Trichosanthis Fructus, Aurantii Fructus Immaturus, Platycodonis Radix, Forsythiae Fructus, 
Stir-baked Descurainiae Semen Lepidii Semen and Stir-baked Raphani Semen. The high content compounds of the above Chinese medicinal pieces included 14 compounds in Table 3, from which 11 compounds were chosen because of consistent tastes with the main tastes of their respective originated Chinese medicinal pieces, and these compounds were more likely to be the quality markers of XXZOL. Trigonelline, malic acid, citric acid, and caffeic acid were the potential sour material bases of XXZOL. Mannitol was the potential sweet material basis of XXZOL. L-phenylalanine, sinapine, forsythoside I, pinoresinol4 -O- $\beta$-D-glucopyranoside, naringin, and neohesperidin were the potential bitter material bases of XXZOL.

\section{Conclusions}

In this work, 15 high content compounds in Xiao'er Xiaoji Zhike oral liquid were identified according to the high-resolution mass data and the MS/MS fragments. The e-tongue collected the sour, bitter, sweet, pungent, and salty samples, which were $1.0 \mathrm{mg} / \mathrm{mL}$ solutions of the reference components with above tastes, and DFA model to recognize the taste characteristics of 23 liquids samples was established to predict the tastes of 15 main components in XXZOL, and the accurate recognition rate was $73.33 \%$, the false rate was $6.67 \%$, and the unrecognized rate was $20 \%$. The concordance rate of their authentic tastes with the tastes of their respective originated Chinese medicinal pieces was $78.57 \%$, and 11 compounds were chosen because of consistent tastes with the main tastes of their respective originated Chinese medicinal pieces. Trigonelline, malic acid, citric acid, and caffeic acid were the potential sour material bases of XXZOL. Mannitol was the potential sweet material basis of XXZOL. L-phenylalanine, sinapine, forsythoside I, pinoresinol4 -O- $\beta$-D-glucopyranoside, naringin, and neohesperidin were the potential bitter material bases of XXZOL. Starting with the taste prediction and confirmation of the main components in XXZOL, this paper provided a method for determining potential quality markers based on the concordant tastes of compounds with their respective originated Chinese medicinal pieces, which provided a scientific basis for the quality evaluation of XXZOL.

\section{Acknowledgements}

The authors appreciate the financial support by the National Key Research and Development Program of China (Grant No. 2019YFC1711201).

\section{References}

Banerjee, M. B., Roy, R. B., Tudu, B., Bandyopadhyay, R., \& Bhattacharyya, N. (2019). Black tea classification employing feature fusion of E-Nose and E-Tongue responses. Journal of Food Engineering, 244, 55-63. http://dx.doi.org/10.1016/j.jfoodeng.2018.09.022.

Bi, S., Xie, R. N., Jin, C. S., Ma, K., Zhang, Y. Q., \& Li, T. S. (2020). Changes of processing process of Aconiti Radix Cocta based on bionic technology. Chinese Traditional and Herbal Drugs, 51(23), 5956-5962.

China, Ministry of Public Health of the People's Republic of China. (2020). Chinese pharmacopoeia (2020 Version a). Beijing: Chinese Pharmaceutical Technology Press.
Ciosek, P., \& Wróblewski, W. (2011). Potentiometric electronic tongues for foodstuff and biosample recognition: an overview. Sensors, 11(5), 4688-4701. http://dx.doi.org/10.3390/s110504688. PMid:22163870.

Feng, B., Wu, Z. F., He, J., Lan, Y., Wang, X. P., Han, X., Jiang, H., Lin, J. Z., Xu, R. C., Zhang, D. K., Zhang, Y., \& Han, L. (2019). A novel bitter masking approach: Powder coating technology-take Sanhuang tablets as an example. Journal of Drug Delivery Science and Technology, 52, 46-54. http://dx.doi.org/10.1016/j.jddst.2019.04.028.

Gan, Y., Hu, N., He, C. J., Zhou, S. Q., Tu, J. W., Liang, T., Pan, Y. X., Kirsanov, D., Legin, A., Wan, H., \& Wang, P. (2019). MnO nanosheets as the biomimetic oxidase for rapid and sensitive oxalate detection combining with bionic E-eye. Biosensors \& Bioelectronics, 130, 254261. http://dx.doi.org/10.1016/j.bios.2019.01.026. PMid:30771714.

Ghasemi-Varnamkhasti, M., Apetrei, C., Lozano, J., \& Anyogu, A. (2018). Potential use of electronic noses, electronic tongues and biosensors as multisensor systems for spoilage examination in foods. Trends in Food Science \& Technology, 80, 71-92. http://dx.doi.org/10.1016/j. tifs.2018.07.018.

Guedes, M. D. V., Marques, M. S., Guedes, P. C., Contri, R. V., \& Kulkamp Guerreiro, I. C. (2021). The use of electronic tongue and sensory panel on taste evaluation of pediatric medicines: a systematic review. Pharmaceutical Development and Technology, 26(2), 119-137. http://dx.doi.org/10.1080/10837450.2020.1860088. PMid:33274664.

Jiang, H. Y., Zhang, M., Bhandari, B., \& Adhikari, B. (2018). Application of electronic tongue for fresh foods quality evaluation: a review. Food Reviews International, 34(8), 746-769. http://dx.doi.org/10.1 080/87559129.2018.1424184.

Legin, A., Rudnitskaya, A., Vlasov, Y., Di Natale, C., Mazzone, E., \& D’Amico, A. (2000). Application of electronic tongue for qualitative and quantitative analysis of complex liquid media. Sensors and Actuators. B, Chemical, 65(1-3), 232-234. http://dx.doi.org/10.1016/ S0925-4005(99)00324-X.

Liang, G., Chen, L., Zhou, Y. C., \& Zhao, F. L. (2018). Effects of Xiaoji Zhike oral solution on symptoms improvement and humoral immunity in children with chronic cough. World Chinese Medicine, 13(12), 3043-3045. https://dx.doi.org/10.3969/j.issn.1673-7202.2018.12.022.

Lin, Z. Z., Zhang, Q., Liu, R. X., Gao, X. J., Zhang, L., Kang, B. Y., Shi, J. H., Wu, Z. D., Gui, X. J., \& Li, X. L. (2016). Evaluation of the bitterness of traditional chinese medicines using an E-tongue coupled with a robust partial least squares regression method. Sensors, 16(2), 151. http://dx.doi.org/10.3390/s16020151. PMid:26821026.

Magro, C., Mateus, E. P., Raposo, M., \& Ribeiro, A. B. (2018). Overview of electronic tongue sensing in environmental aqueous matrices: potential for monitoring emerging organic contaminants. Environmental Reviews, 27(2), 202-214. http://dx.doi.org/10.1139/er-2018-0019.

Mohd Ali, M., Hashim, N., Abd Aziz, S., \& Lasekan, O. (2020). Principles and recent advances in electronic nose for quality inspection of agricultural and food products. Trends in Food Science \& Technology, 99, 1-10. http://dx.doi.org/10.1016/j.tifs.2020.02.028.

Nategh, N. A., Dalvand, M. J., \& Anvar, A. (2021). Detection of toxic and non-toxic sweet cherries at different degrees of maturity using an electronic nose. Journal of Food Measurement and Characterization, 15(2), 1213-1224. http://dx.doi.org/10.1007/s11694-020-00724-6.

Orlandi, G., Calvini, R., Foca, G., Pigani, L., Vasile Simone, G., \& Ulrici, A. (2019). Data fusion of electronic eye and electronic tongue signals to monitor grape ripening. Talanta, 195, 181-189. http://dx.doi. org/10.1016/j.talanta.2018.11.046. PMid:30625530.

Orlandi, G., Calvini, R., Pigani, L., Foca, G., Vasile Simone, G., Antonelli, A., \& Ulrici, A. (2018). Electronic eye for the prediction of parameters related to grape ripening. Talanta, 186, 381-388. http://dx.doi. org/10.1016/j.talanta.2018.04.076. PMid:29784377. 
Pandurangan, M., \& Hwang, I. (2015). Systemic mechanism of taste, flavour and palatability in brain. Applied Biochemistry and Biotechnology, 175(6), 3133-3147. http://dx.doi.org/10.1007/s12010-015-1488-3. PMid:25733187.

Pascual, L., Gras, M., Vidal-Brotóns, D., Alcañiz, M., Martínez-Máñez, R., \& Ros-Lis, J. V. (2018). A voltammetric e-tongue tool for the emulation of the sensorial analysis and the discrimination of vegetal milks. Sensors and Actuators. B, Chemical, 270, 231-238. http:// dx.doi.org/10.1016/j.snb.2018.04.151.

Peris, M., \& Escuder-Gilabert, L. (2016). Electronic noses and tongues to assess food authenticity and adulteration. Trends in Food Science \& Technology, 58, 40-54. http://dx.doi.org/10.1016/j.tifs.2016.10.014.

Shi, Q. R., Guo, T. T., Yin, T. J., Wang, Z. Q., Li, C. H., Sun, X., Guo, Y. M., \& Yuan, W. H. (2018). Classification of pericarpium citri reticulatae of different ages by using a voltammetric electronic tongue system. International Journal of Electrochemical Science, 13(12), 11359-11374. http://dx.doi.org/10.20964/2018.12.45.

Shimizu, F. M., Braunger, M. L., \& Riul, A. (2019). Heavy metal/toxins detection using electronic tongues. Chemosensors, 7(3), 36. http:// dx.doi.org/10.3390/chemosensors7030036.

Toko, K. (1998). Electronic tongue. Biosensors \& Bioelectronics, 13(6), 701-709. http://dx.doi.org/10.1016/S0956-5663(98)00025-6. PMid:9828364.

Wadehra, A., \& Patil, P. S. (2016). Application of Electronic Tongue in Food Processing. Analytical Methods, 8(3), 474-480. http://dx.doi. org/10.1039/C5AY02724A.

Wasilewski, T., Migoń, D., Gębicki, J., \& Kamysz, W. (2019). Critical review of electronic nose and tongue instruments prospects in pharmaceutical analysis. Analytica Chimica Acta, 1077, 14-29. http:// dx.doi.org/10.1016/j.aca.2019.05.024. PMid:31307702.

Wei, Z. B., Yang, Y. N., Wang, J., Zhang, W. L., \& Ren, Q. F. (2018). The measurement principles, working parameters and configurations of voltammetric electronic tongues and its applications for foodstuff analysis. Journal of Food Engineering, 217, 75-92. http://dx.doi. org/10.1016/j.jfoodeng.2017.08.005.

Wu, H. S., Zhang, D. Y., Kang, T. G., \& Zhang, H. (2018). Discrimination of Schisandra sphenanthera fruit and Schisandra chinensis fruit and their origins based on electronic tongue. Zhong Yao Cai, 41(04), 822-828. http://dx.doi.org/10.13863/j.issn1001-4454.2018.04.010.

Xu, M., Wang, J., \& Zhu, L. Y. (2019). The qualitative and quantitative assessment of tea quality based on E-nose, E-tongue and E-eye combined with chemometrics. Food Chemistry, 289, 482-489. http:// dx.doi.org/10.1016/j.foodchem.2019.03.080. PMid:30955639.

Zhang, G. M., Huang, Z. Y., Sun, R., Ye, S. L., \& Feng, Q. (2020a). Xiaoer Xiaoji Zhike oral liquid combined with azithromycin for mycoplasma pneumoniae pneumonia in children: a systematic review and meta-analysis. Evidence-Based Complementary and Alternative Medicine, 2020, 9740841. http://dx.doi.org/10.1155/2020/9740841. PMid:32765636.

Zhang, S. Y., Li, S. B., Xia, Z. Z. L., \& Cai, K. Y. (2020b). A review of electronic skin: soft electronics and sensors for human health. Journal of Materials Chemistry. B, Materials for Biology and Medicine, 8(5), 852-862. http://dx.doi.org/10.1039/C9TB02531F. PMid:31942905.

Zhou, X., Ge, C. X., Chen, Y., Qian, X. J., \& Wang, Y. L. (2020). Clinical effects of Xiaoer Xiaoji oral liquid combined with western medicine in the treatment of children's bronchial asthma. World Chinese Medicine, 15(11), 1611-1614. http://dx.doi.org/10.3969/j.issn.16737202.2020.11.020. 